\title{
Erratum to: Ferroelectric, Thermal, and Magnetic Characteristics of Praseodymium Malonate Hexahydrate Crystals
}

\author{
NAZIR AHMAD, ${ }^{1,3}$ M.M. AHMAD, ${ }^{1}$ and P.N. KOTRU ${ }^{2,4}$ \\ 1.-Condensed Matter Physics Laboratory, Department of Physics, National Institute of Tech- \\ nology, Hazratbal, Srinagar 190006, Jammu and Kashmir, India. 2.-Present address: Crystal \\ Growth and Materials Research Laboratory, Department of Physics and Electronics, University of \\ Jammu, Jammu 180006, India. 3.—e-mail: nazir_16phd11@nitsri.net. 4.—e-mail: pnkotruphy@ \\ gmail.com
}

\section{Erratum to: Journal of ELECTRONIC}

MATERIALS, Vol. 45, No. 4, 2016, pp. 2206-2215

DOI: $10.1007 / \mathbf{s 1 1 6 6 4 - 0 1 5 - 4 2 6 9 - 4}$

Nazir Ahmad has been added as a corresponding author as shown in this erratum.

The correct captions for Figs. 5 and 8 are as follows:

Fig. 5. Plot of $\ln \left(1 / \varepsilon_{\mathrm{r}}-1 / \varepsilon_{\max }\right)$ versus $\ln \left(T-T_{\mathrm{c}}\right)$ for single crystal of $\mathrm{PMH}$.

Fig. $8 . \ln \left(1 / \varepsilon_{\mathrm{r}}-1 / \varepsilon_{\text {max }}\right)$ versus $\ln \left(T-T_{\mathrm{c}}\right)$ for pellet sample. 